\title{
A New Spectral Analysis Algorithm for SAR Data Processing of ScanSAR Data and Medium Resolution Data without Interpolation
}

\author{
Ana Vidal-Pantaleoni, Miguel Ferrando \\ Departamento de Comunicaciones \\ Universidad Politécnica de Valencia, Camino de Vera s/n, E-46071 Valencia, Spain \\ T: +34-963879710, F: +34-963877309, E-mail: avidal@dcom.upv.es
}

\begin{abstract}
This paper describes a new approach for ScanSAR data image generation, which performs efficient data processing and multilook operation without the need of interpolation stages. This new technique is based on the Spectral Analysis method (SPECAN) combined with the well-known Chirp Z-Transform. In this paper, it is also shown that this new method is very efficient for obtaining near real-time images for browsing or on-board burst mode image generation overcoming classical problems of standard SPECAN method.
\end{abstract}

\section{INTRODUCTION}

A new trend in Synthetic Aperture Radar (SAR) is the deployment of complex sensors capable of providing a wider swath by means of electronic beam steering. This type of instrument is called Scanning SAR (ScanSAR). It has become one of the most promising techniques for Earth global monitoring at microwave frequencies. The data produced by this type of system have some features that require the design of special SAR processing algorithms. For that reason, former algorithms should be updated in order to cope efficiently with the new scenario.

In this context, medium Resolution SAR brings promising possibilities for satisfying near real-time SAR data processing. The most efficient method in this framework is the Spectral Analysis method (SPECAN) as described in [1]. On the other hand, it presents serious drawbacks concerning decimation like for instance the need of interpolation stages. Interpolation is a time-consuming and inaccurate procedure and it must be avoided when possible.

In this paper a new method with a modified SPECAN approach based on the Chirp Z-Transform is presented. It is shown to provide an elegant way for overcoming the traditional problems of SPECAN at the expenses of increasing the computational cost. However, it provides a product which requires no further interpolation when a reduced resolution image is desired.

\section{SCANSAR SYSTEM PARAMETERS}

The ScanSAR instrument takes $N_{d}$ echo responses in one swath during the dwell time $T_{d}$. Then, it focuses to the adjacent swath and does the same procedure until it reaches the last swath. It returns to the same swath after the return time $T_{R}$, which is obviously the same for all swaths. $T_{R}$ is the sum of all dwell times plus the time to setup the scanning system. In general, each swath uses a different Pulse Repetition Frequency (PRF). The number of looks of the system comes from the redundancy of data in Doppler spectrum. The antenna illumination times from different bursts overlap, giving a redundancy which corresponds to the natural number of looks of the system. Extra looks could be extracted by data processing.

Classical full resolution SAR algorithms can be adapted to ScanSAR data. These algorithms bring a product that is fully compatible with the normal mode SAR images. However, some additional stages must be introduced in the processing chain like zero-padding and filtering. Moreover, zero padding stages do not cope with the fact that the return time is not generally a multiple of the inverse of the sampling frequency. If that fact is ignored, a perturbation factor is introduced in the system.

\section{SPECAN ALGORITHM}

SPECAN technique is more logically oriented to process ScanSAR data since it processes bursts independently. SPECAN is an efficient technique for compression of quadratic phase (Chirp) signals. It is a technique originally developed in [1] for normal mode SAR but it suits very well to ScanSAR data because of the processing of the signal in bursts. If the principle of stationary phase applies, time may be latched in a unique way with frequency. The special nature of the Chirp signals, joined to the Convolution Theorem, allow us to perform Chirp decorrelation through a mixing and a unique Fast Fourier Transform (FFT) as indicated in Fig. 1. The mixing is equivalent to decorrelation in frequency and since frequencies are linked to time via the stationary phase principle, then the decorrelated signal is obtained in the spectral domain with a scaling factor.

The algorithm can not easily cope with range curvature correction. Therefore, no range migration correction is considered in the following assuming that the decimation performed in the image makes the range migration to affect less than one pixel cell. This assumption is valid for $\mathrm{C}$-Band sensors like next ENVISAT ASAR. The Doppler phase 
history must be well approximated by a quadratic model, which is normally the case with low squint angles.

SPECAN technique can also be applied to chirp pulse compression. For both range and azimuth processing, the sampling frequency is represented by $F_{\mathrm{S}}$, the Frequency Modulation rate by $F_{R}$ and the Chirp signal is $h_{S}(t)$ as described in (1).

$$
h_{S}(t)=\exp \left(j 2 \pi \frac{F_{R}}{2} t^{2}\right)
$$

The previous description is based on the use of continuous signals in time, but SAR signal comes in digitized form and all the processing is performed by digital processing methods. The relation between number of samples in time and in frequency is determined by the FFT operation as

$$
\Delta k=\Delta n \frac{F r}{(F s)^{2}} N_{H F T}
$$

where $k$ is the discrete frequency sampling, $n$ the discrete time sampling and $\mathrm{N}_{\mathrm{FFT}}$ the FFT length. The decimation ratio between input raw data and output image is determined by the processing parameters, but the number of samples must be always an integer. For that reason, resampling is introduced in the system for multilook and tiling operations.

When considering SPECAN ScanSAR processing, the algorithm has to deal with fixed return time and different sampling frequencies for different swaths. Besides that, the FM rate in azimuth changes slightly with the orbit characteristics. For those reasons, the decimation ratio should be externally adapted by interpolation or by scaling as described in [3].

\section{NEW CZT-SPECAN ALGORITHM}

Problem description

Final pixel spacing in the deramp-FFT method is restricted by the FM rate, sampling frequency and FFT size. It does not bring a satisfactory solution for ScanSAR data because in this case the system has less degrees of freedom for choosing the correct processing parameters. That is, even with a proper selection of FFT size, algorithms should make use of interpolation in order to get samples at the appropriate frequency positions. In range compression, it is also interesting to get an algorithm with freely selectable decimation factor in order to obtain a uniform image from the different swaths.

\section{Algorithm Description}

The new algorithm has been designed to bring a simple solution. It is based on SPECAN implementing spectral analysis by the Chirp Z-Transform (CZT). Deramping is
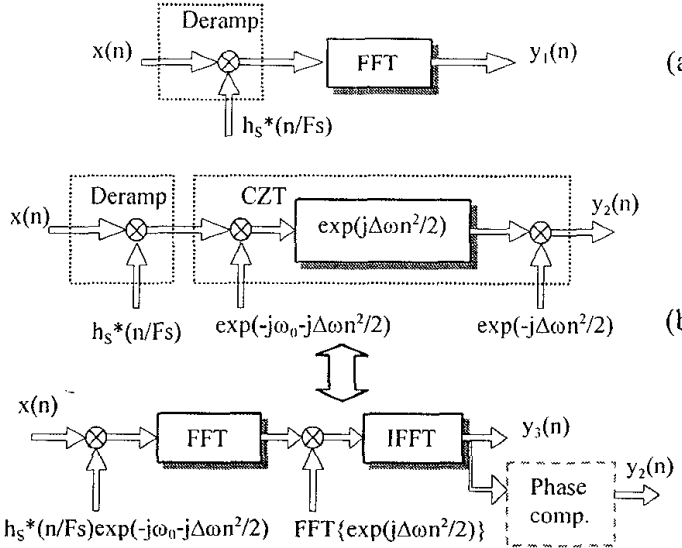

Fig. 1. (a) SPECAN, (b) CZT-SPECAN block diagram

performed first, centering the spectrum to the Doppler Centroid frequency.

The Chirp Z-Transform performs spectrum estimation and spectrum scaling in a similar way to the scaling functions in the Chirp Scaling Algorithm as described in [3]. The main difference yields in the fact that the SPECAN Z-Chirp Transform algorithm (CZT-SPECAN) performs scaling in the last stage of the processing chain. CZT involves a convolution that it is implemented by fast frequency convolution as shown in Fig. 1. The CZT provides spectrum contents at equally spaced positions beginning at an arbitrary point. The initial frequency is $\omega_{0}$ and the spectral separation is $\Delta \omega$ as described in [2]. The deramping operation is joined to the first complex multiplication of the CZT. The last complex multiplication may be avoided if no phase preservation is needed, which is often the case in medium resolution processing. When the phase wants to be preserved, an extra
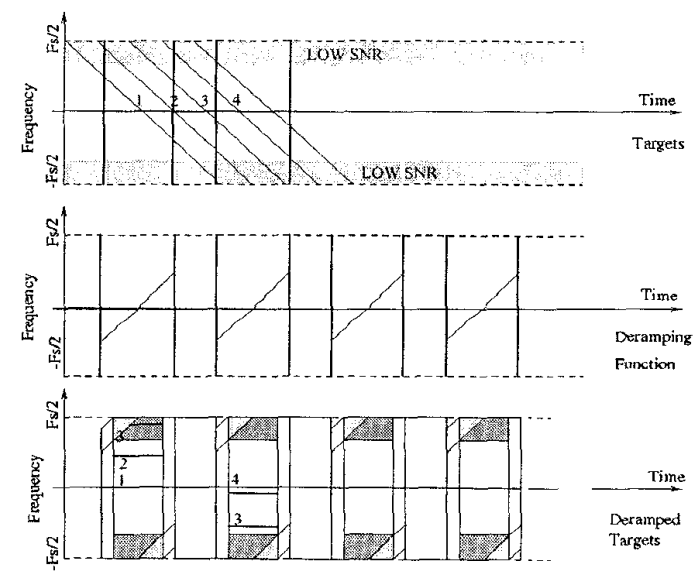

Fig. 2. Deramp and multilook strategy 
complex multiplication must be added to the processing chain. It includes the last CZT complex multiplication and the phase compensation of the SPECAN technique.

The selection of the spectral zone to use is done by a new strategy described in Fig. 2. The spectrum is centered on zero Doppler frequency and the region with highest Signal to Noise Ratio is chosen. By doing so, the effect of scalloping is reduced and no further correction is needed. The extent of the spectral region is different for each swath and it is adapted to correct FM rate variations. Once the spectral window is chosen, the spectral content at arbitrary positions is extracted by the Chirp Z-Transform algorithm. No radiometric correction has been employed because the multilook strategy reduces the scalloping effect, as it is shown below with the simulation work.

In summary, the FFT of standard SPECAN algorithm is turned into two FFT's and a complex multiplication as it is shown in Fig. 1. The advantage over other Fast Frequency Range-Doppler methods described in [4] is that the FFT length is short in both cases and it can provide an efficient solution for reduced resolution ScanSAR data processing.

\section{ALGORITHM PERFORMANCE}

The new technique has been tested over ERS-1 data on the area of Flevoland test site. The ERS-1 data has been converted to burst mode rejecting a number of range lines. The selected ScanSAR parameters are similar to those of first swath of ENVISAT ASAR wide swath mode. Return time of $0.156667 \mathrm{sec}$. and 39 echoes per burst have been processed for the image displayed in Fig. 3. The same constraints as ScanSAR data have been applied to the problem. The image has been processed with the CZT-SPECAN in both range and azimuth. It presents a pixel spacing of $80 \mathrm{~m}$ in azimuth and $100 \mathrm{~m}$ in range and it has been processed with 12 looks. These are 3 looks in azimuth (system looks) and 4 processing looks in range. From the analysis of a uniform window in the sea, the equivalent number of looks measured is equivalent to

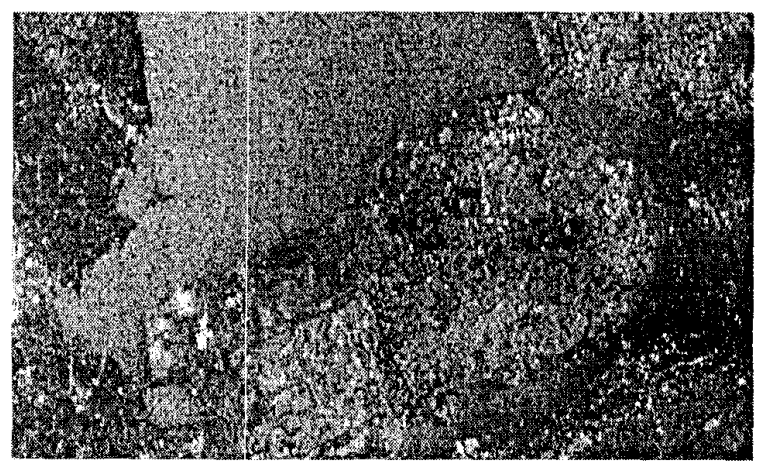

Fig. 3. CZT-SPECAN Flevoland the real figure. A synthetic ScanSAR point target has been processed and its results also show a good behavior of the algorithm.

The computational cost increases with regard to standard SPECAN, since a FFT and a complex multiplication are introduced in the system. Due to such fact, this technique increases complexity over SPECAN but gives more flexibility to the final image pixel spacing without requiring interpolation. The introduction of new stages, however, is produced on the burst data and FFT lengths are short.

\section{CONCLUSIONS}

A new method for performing medium resolution ScanSAR data processing without interpolation has been presented. It is based on deramping and spectral analysis through the Chirp Z-Transform that is performed by Fast Frequency Convolution. This new approach simplifies the multilooking stages and provides a uniform image in all swaths. The algorithm is capable of providing uniform pixel spacing independently of the PRF or the FM rate with respect to azimuth processing, and the same can be said with regard to range processing.

This new algorithm has important advantages over the existing standard SPECAN, although the overall number of operations is increased. The most important feature, however, is that interpolation stages are not necessary for the multilook operation. A new multilook strategy that reduces the scalloping effect has been proved.

\section{ACKNOWLEDGMENTS}

The authors thank the European Space Agency (Data Compression Workgroup) for providing the raw ERS-1 data.

\section{REFERENCES}

[1] M. Sack, M. R. Ito and I. G. Cumming, "Application of efficient linear FM matched filtering algorithms to synthetic aperture radar processing", IEE Proc. Pt. F, vol. 132, No. I, pp. 45-57, February 1985.

[2] A. V. Oppenheim and R. W. Schafer, Discrete-Time Signal Processing, Englewood Cliffs: Prentice Hall, 1989 , pp. 623-628.

[3] A. Moreira, J. Mittermayer and R. Scheiber, "Extended Chirp Scaling Algorithm for Air- and Spaceborne SAR Data Processing in Stripmap and ScanSAR Mode", IEEE Trans. on Geosci. Remote Sensing, vol. 34, pp II23$1136,1996$.

[4] I. Cumming, Y Guo and F. Wong, "A Comparison of Phase Preserving Algorithms for Burst-Mode SAR Data Processing", in Proc. IGARSS'97, 1997. 